\title{
New CoenzymeQ10 Formulations Increase Mental Concentration and Focused Attention Related to ATP Production
}

\section{Enno Freye ${ }^{1^{*}}$ and Hans-Peter Strobel ${ }^{2}$}

${ }^{1}$ Heinrich-Heine-University Düsseldorf, Universittässtrasse, Germany

${ }^{2}$ Department of Galenics, Energy development Ltd, Davos-Platz, Switzerland

*Corresponding author: Freye E, Heinrich-Heine-University Düsseldorf, Universittässtrasse, Germany, Tel: 0041-77454-2477; E-mail: enno.freye@uni-duesseldoerf.de Received: May 24, 2018; Accepted: May 31, 2018; Published: June 05, 2018

Copyright: (c) 2018 Freye E, et al. This is an open-access article distributed under the terms of the Creative Commons Attribution License, which permits unrestricted use, distribution, and reproduction in any medium, provided the original author and source are credited.

\begin{abstract}
Objectives

Two new CoQ10 preparations are on the market, of which however, very little is known about their ability to increase ATP production, the final end product in mitochondrial activity. We therefore set out to measure ATP synthesis in granulocytes of volunteers before and after a five-week administration of either Greenspeed ${ }^{8}$ or Q10 Revolution and sought to determine if this is reflected in some way to an increase in concentration and vigilance.

\section{Methods}

12 young healthy volunteers (mean age 21, 10 males, two females) were given either one of the two CoQ10 supplements over a period of 5 weeks in a cross-over randomized double-blind fashion. ATP concentration was measured in granulocytes before and after five weeks, using bioluminescence luciferase probes. In addition, the $\mathrm{d} 2$ concentration and stress test was used to determine mental concentration before and after each of the Q10 formulations.

\section{Results}

The two Q10 formulations (Greenspeed and Q10 Revolution ${ }^{\circ}$ ) increased the formation of ATP within the granulocytes following intake over of five-week period. This increase was highly significant following both formulations $(\mathrm{p}<0.0005)$, while at the same time it increased concentration and mental performance resulting in a significant reduction $(\mathrm{p}<0.001)$ of mistakes within the $\mathrm{d} 2$-concentration and stress test. The latter was closely correlated with ATP increase resulting in a correlation coefficient of $\mathrm{r}^{2}=0.84$ for both formulations.

\section{Conclusion}

The two CoQ10 formulations (Q10 Revolution and Greenspeed ${ }^{\circ}$ ) on the European market demonstrate significant increase in ATP synthesis within mitochondria, while at the same time increase mental performance considerably. Both formulations use different strategies of how to increase Q10 getting into the cell since plasma concentrations by their own do not properly reflect ATP synthesis within mitochondria.
\end{abstract}

Keywords: CoQ10; Q10 formulation; Bioluminescence luciferase; ATP synthesis; Greenspeed ${ }^{\circ}$ Q Q10 Revolution; ${ }^{\circ}$ 2 Concentration and stress test

\section{Introduction}

While it is generally accepted that Q10 is a necessary component in normal function within the electrical transport chain (ETC) of mitochondria, little is known in regard to the alleged increase in adenosine triphosphate (ATP) synthesis following supplementation of Q10. This seems of importance as to days nutrition is devoid of sufficient Q10 [1], while on the other hand there is a decline of biosynthesis of Q10 with age [2,3]. Such decline in Q10 biosynthesis is even more pronounced once people are under statin medication, a compound that actively inhibits the body's own synthesis [4], resulting in a number of ailments starting from early brain decline with dementia [5], to diabetes [6], even inducing arteriosclerosis [7] to progressive cardiovascular disease with stroke [8] and/or heart failure [7]. Thus, a Q10 supplements may become a necessity in the elderly as well as in people taking cholesterol lowering medication in order to ensure sufficient organ function.

And although a number of Q10 supplements are on the market all of which underline their superior bioavailability [9], nothing is being mentioned in regard to their ability to boost the electrical transport mechanism within mitochondria resulting in an increase in ATP synthesis.

We therefore set out to study the efficacy of two new Q10 formulations on the market (Greenspeed ${ }^{\circ}$ and Q10 Revolution ${ }^{*}$ ) which aside from claiming optimal bioavailability, also suggest optimal 
efficacy in regard to mental performance, concentration and the reversal of chronic fatigue.

\section{Materials and Methods}

12 otherwise healthy athletes who are actively engaged in competition sports ( 10 male and 2 females, mean age $21 \pm 5$ years of age) were given Greenspeed ${ }^{\circ}$ and Q10 Revolution respectively at two different occasions over a period of 5 weeks, 1 ampule of $25 \mathrm{ml}$ per day in a cross-over study design. Aside from different constituents both also contained different amounts of Q10 of $80 \mathrm{mg}$ and $420 \mathrm{mg}$ respectively. Prior to Q10 intake, ATP (adenosine triphosphate) concentrations were measured within isolated granulocytes following venipuncture. For analysis a technique based on bioluminescence through the addition of D-luciferin probes was used, and ATP levels were determined before, and 5 weeks after supplementation of each of the Q10 formulations. In the presence of magnesium, oxygen and ATP, the protein luciferase catalyzed oxidation of the substrate luciferin, which was associated with light emission which then was measured and correlated with a standard curve of ATP concentration (Biovis Diagnostics Ltd, Limburg, Germany). Using this strategy, the luciferin targeted the mitochondrial matrix and the outer surface of the plasma membrane, a technique which is described in detail elsewhere [10,11]. For monitoring of ATP formation and functionality of the mitochondrial matrix and the peri-cellular space in living cells, the overall procedure within the study was broken down into two different steps

- Measurement of the intracellular in-vitro ATP-profile before, and five weeks after intake of the Q10 formulation.

- Following a wash-out period of 5 weeks the same subjects were given the other Q10 formulation in a cross-over design over a period of 5 weeks. Thus, each individual took part on four separate occasions determining the ATP concentration before and after Q10 intake.

At the same instances, subjects underwent the concentration and attention test (d2-test), which is described in detail elsewhere [12]. In short, the test consisted in stroking off the selective letter "d" with two bars on the right-hand side of randomly assigned 21 targets in 14 long rows of letters of the alphabet resulting in a detail-discrimination within a specific time period of $3 \mathrm{~min}$. Thereafter hits, misses and mistakes were calculated and computed as per cent of what should have been done correctly.

\section{Composition of the two Q10 formulations}

- Greenspeed (from Energy Development, Davos-Platz/ Switzerland) is an oral solution, where the recommended $25 \mathrm{ml}$ contained the following ingredients and dosages: ubiquinone (or Q10) $80 \mathrm{mg}$, Siberian ginseng extract $300 \mathrm{mg}$, vitamin $\mathrm{B}_{3}$ (nicotinamide) $48 \mathrm{mg}$, vitamin $\mathrm{B}_{2} 4.2 \mathrm{mg}$, vitamin $\mathrm{E} 25 \mathrm{mg}$, vitamin C $160 \mathrm{mg}$, the sugar monosaccharide D-Ribose in a dose of $1000 \mathrm{mg}$, Vit $\mathrm{D}_{3}$ in a dose of $600 \mathrm{IU}$ and passion fruit extract in a dose of $30 \mathrm{mg}$. For better reabsorption of Q10, silymarin $5 \mathrm{mg}$, curcumin $5 \mathrm{mg}$, while the flavonoid quercetin $2 \mathrm{mg}$ was used for antioxidative purposes and chrysin $5 \mathrm{mg}$ was added because of its anti-inflammatory properties. In addition to these additives gum Arabic was used for emulsifying purposes.

- Q10 Revolution (from JAG group Polska, Poland) is also taken as an oral solution, where the recommended $25 \mathrm{ml}$ contained the following ingredients and dosages: ubiquinone (or Q10) in a dose of $420 \mathrm{mg}$, NADH (or Q1) $20 \mathrm{mg}$, vitamin E $60 \mathrm{mg}$, the vitamin Bcomplex and especially $B_{12} 3500 \mu \mathrm{g}$, the sugar D-Ribose $1000 \mathrm{mg}$, Vitamin $D_{3} 20.000$ IU plus evening primrose oil for solubilization and glycerin plus diacetyl for emulsifying purposes.

\section{Statistical analysis}

The number of subjects necessary to demonstrate statistical significance was calculated presuming a $70 \%$ incidence of difference in ATP concentration as demonstrated elsewhere [13]. These results were taken in order to calculate the number of individuals necessary to demonstrate significant difference in ATP increase in subjects with and without the nutraceutical drink following a period of five weeks. Power analysis assumed an at least $30 \%$ increase within mitochondrial ATP synthesis following consumption of each of the energy drinks. With a value of $\alpha=0.05$ and $\beta=0.90$ it was computed that at least 12 subjects were required in order to demonstrate significance. To minimize the effect of data loss a total of 15 volunteers were enrolled.

All statistical analysis was performed using the Prism 5 software for Mac OS X (Graph Pad Software Inc. San Diego, USA). For computation of statistical significance in the concentration and attention test as well as the increase in ATP formation within mitochondria of granulocytes, results following each Q10 formulation were compared with the control phase using the Wilcoxon signed rank test or the paired t-test for statistical difference between the two formulations when indicated, whichever was applicable.

Under the presumption of a non-parametric distribution, computation of a correlation between the numbers of error rates in the d2-test and their corresponding ATP values, the Spearman correlation test was done. All statistical tests were two-sided and were considered as significant at the $\mathrm{p}<0.05$ level.

\section{Results}

Of the originally 15 enrolled subjects, there was a drop out of 3 participants, so at the end 12 participants would finish the study design (mean age $21 \pm 5$, mean weight, $68 \pm 5 \mathrm{~kg}$, mean height $169 \pm 12 \mathrm{~cm}$ ). Starting off with a mean ATP concentration of $210 \mathrm{pmol} / 10^{6}$ cells, Greenspeed $^{\circ}$ was able to increase ATP to a mean of $630 \mathrm{pmol} / 10^{6}$ cells, following intake for 5 weeks ( $<0.0001$, Figure 1 ). Such an increase was also observed after a 5 weeks intake of Q10 Revolution reaching a mean of 580 pmol/106 cells which was significant to control $(\mathrm{p}<0.0005)$, with only a marginal difference $(\mathrm{p}<0.054)$ to the increase in ATP after Greenspeed" (Figure 1).

The $\mathrm{d} 2$ concentration. and stress test was applied at the same instances when ATP levels were determined, and there was a highly significant reduction in the number of mistakes and misses being made by subjects when compared to control. From a mean of $36 \%$ before Greenspeed $^{\circ}$ and $38 \%$ before Q10 Revolution respectively, the incidence of misses and mistakes declined significantly (Figure 2) following both formulations being only 23 per cent and 19 per cent respectively. Between. the two formulations this decline was significant at the $\mathrm{p}<0.05$ level in favor of Greenspeed". 


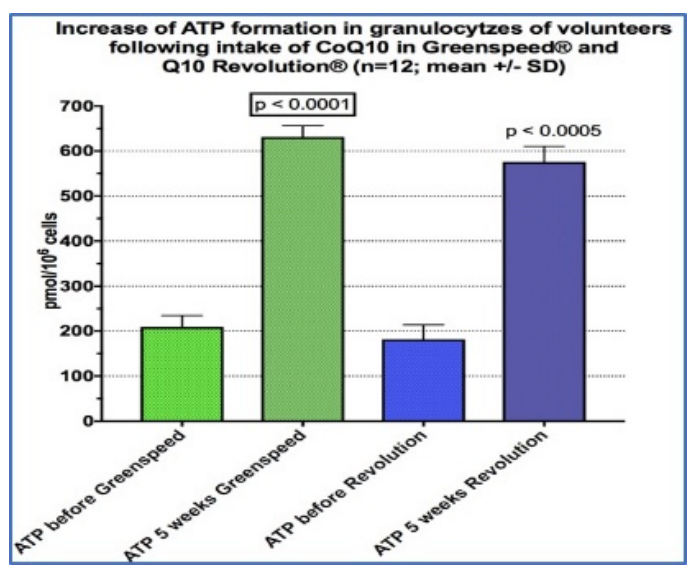

Figure 1: ATP concentration within granulocytes of peripheral blood in volunteers, before and after intake of two different Q10 formulations over a period of 5 weeks.

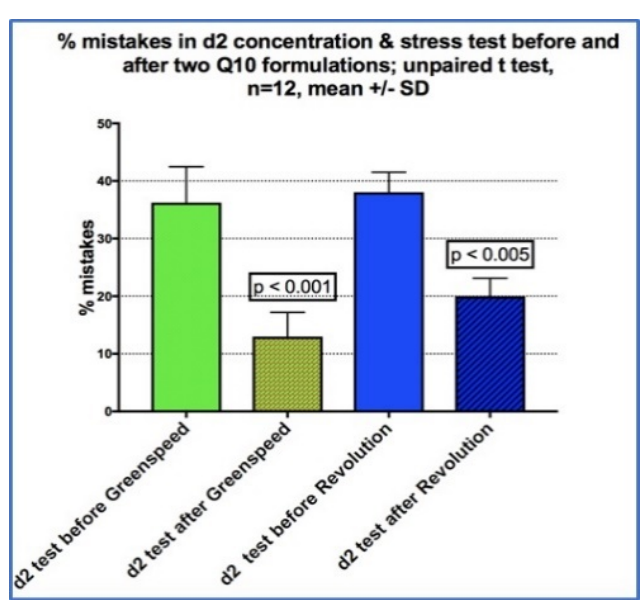

Figure 2: Significant decline in the percentage of misses and mistakes within the $\mathrm{d} 2$ concentration and stress test before and following the intake of two different Q10 formulations, Greenspeed ${ }^{\circ}$ and Q10 Revolution respectively.

In addition, calculation of a possible linear correlation between the ATP levels before and after the intake of both Q10 formulations as well as the reduction. in misses and mistakes in the $\mathrm{d} 2$ - concentration and stress test, reveal a close correlation with a coefficient of $\mathrm{r}^{2}=0.84$ (Figure 3). The latter underlines the presumption that mental performance with focused attention very much is dependent on ATP synthesis within brain cells.

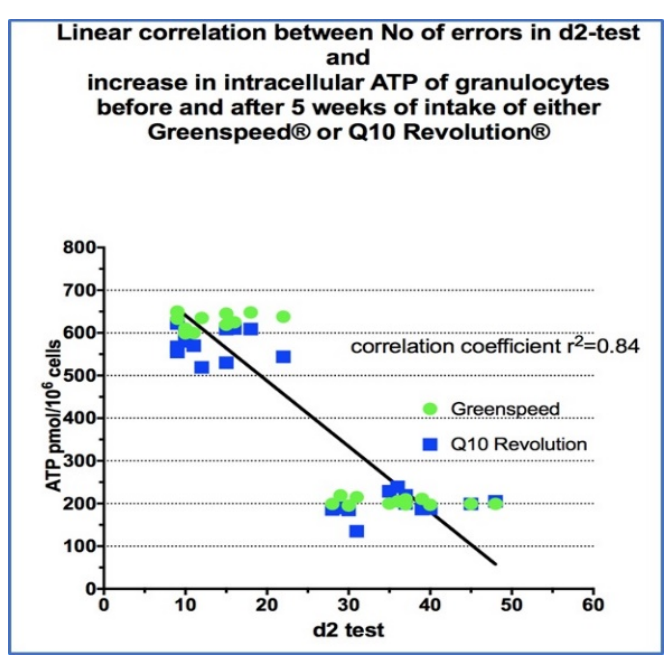

Figure 3: Linear correlation between ATP concentration before and after Q10 supplementation with the results of the $\mathrm{d} 2$-concentration and stress test over a period of 5 weeks, using either the Greenspeed $^{\circ}$ or the Q10 Revolution supplement demonstrating a close correlation with a high coefficient.

\section{Discussion}

First and utmost of all, this to the knowledge of the investigators, is the first time that ATP levels were determined after a Q10 supplementation in humans. Such data are of importance since the ATP level actually presents the net effect of any kind of Q10 formulation being touted as beneficial to boost mitogenesis and mitochondrial function. Such thinking is in contrast to most all other publications on Q10, where bioavailability is considered the important part in a Q10 supplementation [9]. While bioavailability does indeed play an important part of any kind of Q10 supplement, what actually counts is the amount of Q10 that gets into the cell, to the mitochondria resulting in an activation of the electrical transport chain with an increase in the output of ATP.

Such an increase in ATP synthesis indeed could be demonstrated with the two new Q10 formulations. And in spite the lower Q10 content within Greenspeed when compared to Q10 Revolution having a $>5$ fold higher content per vial, both induced ATP synthesis. From such data it can be derived that the amount of Q10 is not the most important vector to boost ATP synthesis but actually how much gets into the cell and to the mitochondrial level, which ultimately will result in an increased synthesis of ATP.

This assumption is underlined by the seemingly importance of the other components within the formulation of Greenspeed', such as the ginseng extract with its active ingredients eleutheroside $\mathrm{A}$ and $\mathrm{E}$, which can be considered as enhancers as they most likely act as PGP-pump inhibitors resulting in a higher concentration of Q10 at the site of action within the cellular matrix. Such connotation is corroborated by data of others demonstrating that the active ingredients in eleutherococcus senticosus selectively inhibit p-glycoprotein drug efflux pump expression of the multidrug transporter P-glycoprotein (PGP) system, which is encoded by the mdr1 gene being an integrated part of pharmacokinetic interactions $[14,15]$. Taken together those natural antioxidants, and anti-inflammatory agents found in 
Page 4 of 4

Greenspeed $^{\circ}$ result in a higher rate of solubilization which in combination with the inhibition of efflux pump of cells lead into higher ATP formation within the neuronal tissue. By using the same formulation and as demonstrated elsewhere. Freye et al. already had demonstrated a higher rate in synthesis of ATP cortical neuronal cells using the electroencephalogram where the higher firing rate of cortical cells was reflected in an increase of EEG-activity within the fast betadomain [13]. Since the firing rate of cortical neuronal cells depends heavily on the synthesis of their burning fuel ATP, EEG-power spectra were considered as reliable indicators of a higher ATP synthesis within neuronal cells following Q10 intake [16]. The other formulation Q10 Revolution however, seems to compensate for such a necessary PGPinhibition by the use of $>5$ fold higher dosage of Q10 in its content. In addition, this formulation contains another necessary component for ATP synthesis, i.e., NADH or Nicotinamide-Adenin-DinucleotidHydrid (often referred to as Coenzyme Q1), which selectively activates the electrical transport chain within the mitochondria acting like an ignition spark on complex I setting off the onset in ATP synthesis [17] while at the same time acting also as a potent antioxidant [18]. As a net outcome, this Q10 formulation also results in a higher rate in the synthesis of ATP within mitochondria when being applied over a period of 5 weeks.

In addition, this higher synthesis in ATP also does effect mental performance and especially focused attention. Both Q10 supplements did induce concentration capabilities as demonstrated in the $\mathrm{d} 2$ concentration and stress test resulting in more focused attention with lesser mistakes and misses. While there is a statistical higher benefit after Greenspeed this however is marginal as it is expected that in the real world both Q10 formulations would conclusively boost mental performance in a similar fashion.

Another important aspect within this study is the fact that ATP concentration within cells and focused attention as being determined in the $\mathrm{d} 2$-concentration and stress test do demonstrate a very close correlation. And although correlation is not causation, the close coefficient of $r^{2}=0.89$ strongly implies that without sufficient ATP synthesis within neuronal cells, there is insufficient function which similarly has been demonstrated in the EEG-power spectra within the electroencephalogram and neuronal cells heavily depend on this elementary energy substrate in. order to function properly.

In conclusion, the present study in human volunteers has conclusively proven the importance of Q10 in neuronal functioning while at the same time demonstrating that the plasma level of Q10 is not the most important vector to boost ATP synthesis. More so, it is important how much of this absorbed Q10 gets into the cell in order to be used by the machinery within the mitochondria.

\section{References}

1. Weber C, Bysted A, Holmer G (1997) The coenzyme Q10 content of the average Danish diet. Int J Vitam Nutr Res 67: 123-129.

2. Hernández-Camacho JD (2018) Coenzyme Q10 Supplementation in Aging and Disease. Front Physiol 9: 44.

3. Gutierrez-Mariscal FM (2018) Coenzyme Q10: From bench to clinic in aging diseases, A translational Review. Crit Rev Food Sci Nutr 16: 1-18.

4. Nawarskas JJ (2005) HMG-CoA reductase inhibitors and coenzyme Q10. Cardio Rev 13: 76-79.

5. Wanamaker BL (2015) Cholesterol, Statins, and Dementia: What the Cardiologist Should Know. Clin Cardiol 38: 243-250.

6. Chogtu BR, Bairy KL (2015) Statin use and risk of diabetes mellitus. World J Diabetes 6: 352-357.

7. Okuyama H (2015) Statins stimulate atherosclerosis and heart failure: pharmacological mechanisms. Expert Rev Clin Pharmacol 8: 189-199.

8. Rundek T (2004) Atorvastatin Decreases the Coenzyme Q10 Level in the Blood of Patients at Risk for Cardiovascular Disease and Stroke. Arch Neurol 61: 889-892.

9. Liu ZX, Artmann C (2009) Relative bioavailability comparison of different coenzyme Q10 formulations with a novel delivery system. Altern Ther Health Med 15: 42-46.

10. Morciano G (2017) Use of luciferase probes to measure ATP in living cells and animals. Nature Proto 12: 1542-1562.

11. Lundin A (2000) Use of firefly luciferase in ATP-related assays of biomass, enzymes, and metabolites. Meth Enzymol 305: 346-370.

12. Brickenbach R (2002) d2 Attention Stress Test. Götingen, Berne, Toronto, Seattle: Hogrefe Verlag 9: 1-4.

13. Freye E, Strobel HP (2016) Changes within the Electroencephalogram and Increase in Mental Concentration are related to Differences in Solubulisation and Composition of Different Q10-Formulations. Nat Prod Chem Res 4: 2 .

14. Choi CH, Kang G, Min YD (2003) Reversal of P-glycoprotein-mediated multidrug resistance by protopanaxatriol ginsenosides from Korean red ginseng. Planta Med 69: 2325-2240.

15. Chen XP (2006) Reversing multidrug resistance by RNA interference through the suppression of MDR1 gene in human hepatoma cells. World J Gastroenterol 12: 3332-3337.

16. Howarth C, Peppiatt-Wildman CM, Attwell D (2010) The energy use associated with neural computation in the cerebellum. J Cereb Blood Flow Metab 30: 403-414.

17. Gemperli A, Dimroth CP, Steuber J (2003) Sodium ion cycling mediates energy coupling between complex I and ATP synthase. PNAS 100: 839-844

18. Kirsch M, De Groot $\mathrm{H}(2001) \mathrm{NAD}(\mathrm{P}) \mathrm{H}$, a directly operating antioxidant. FASEB J 15: 1569-1574. 